\title{
Prediction Algorithm of Malignant Ventricular Arrhythmia Validated Across Multiple Online Public Databases
}

\author{
Wei Wei Heng ${ }^{1}$, Eileen Su Lee Ming ${ }^{1}$, Ahmad Nizar B Jamaluddin ${ }^{2}$, Fauzan Khairi Che Harun ${ }^{1}$, \\ Nurul Ashikin Abdul-Kadir ${ }^{1}$, Che Fai Yeong ${ }^{1}$ \\ ${ }^{1}$ Universiti Teknologi Malaysia, Johor, Malaysia \\ ${ }^{2}$ Ara Damansara Medical Centre, Selangor, Malaysia
}

\begin{abstract}
Prediction of malignant ventricular arrhythmia ( $m V A)$ is essential to prevent sudden cardiac death. There were mainly three research clusters on $m V A$ prediction using electrocardiogram (ECG): prediction using CUDB, $S D D B$ and private databases. Comparability and generalization issue arose due to the different usage of arrhythmic datasets for analysis. Very few studies attempted short-term prediction of $m V A$ using multiple databases, and those studies achieved low prediction performance. Our study aims to improve the prediction performance involving multiple databases and to promote the algorithm comparability by performing more comprehensive comparability study while including a more complete set of data available from the public databases. In our study, eight statistical box count features derived from phase space reconstruction on ECG signal were classified using maximum thresholding method. This was followed by performance benchmarking against the first two clusters of existing research and a performance evaluation using the combined set of databases. Our algorithm using box count coefficient of mean absolute deviation achieved over 90\% of accuracy and over 4-minutes prediction time for all the three set of performance evaluations. This algorithm outperforms the existing work by introducing lower computational efforts.
\end{abstract}

\section{Introduction}

More than half of the sudden cardiac deaths are attributed to malignant ventricular arrhythmia (mVA) (1) which refers to a group of life-threatening ventricular arrhythmia comprising of ventricular fibrillation (VF) and ventricular tachycardia (VT). It is vitally important for patient to receive prompt medical intervention before mVA occurs. Based on the literature review, there were three different clusters of research on mVA prediction using ECG which, in the authors' point of view, could be categorized according to the arrhythmic database used for their analysis. The prediction works were grouped into prediction using Creighton University Ventricular Tachyarrhythmia Database (CUDB) (2,3), Sudden Cardiac Death Holter Database (SDDB) $(4,5)$ or MITBIH Malignant Ventricular Arrhythmia Database (VFDB) and private database $(6,7)$.

The comparability and generalization issue arose due to the different usage of arrhythmic data sets for analysis, including different public databases, local databases which are not publicly available $(6,7)$ and incomplete data set from certain databases $(2,8)$. Different databases might come from different population with varying underlying diseases, age, gender distribution and so on, resulting in different independent variables for the ECG signal. Using only partial data set from certain databases tends to result in more optimistic performance measure because the excluded data sets might decrease the performance significantly if tested.

Meanwhile, different feature extraction methods and classifiers were used in various previous investigations. Fair comparison of the effectiveness of each mVA prediction techniques could barely be made and generalization capability of each algorithm needs further investigation through re-implementation and reevaluation. One research work attempting to compare short term prediction of mVA using multiple major databases (9), including 24 CUDB recordings, 22 VFDB recordings, 13 MIT-BIH Arrhythmia Database (MITDB) recordings and three MIT-BIH Normal Sinus Rhythm Database (NSRDB) recordings, did not show favourable results. The prediction was done by investigating the difference of power spectral density of signals using regularized least square. The accuracy was only $37.5 \%$ on CUDB and $36.4 \%$ on VFDB. This demonstrated both the possibility and potential problems of applying a technique across multiple databases.

Our study aims to provide a prediction algorithm, with high accuracy and long prediction interval, that is validated across all major databases besides enhancing the algorithm comparability among different research clusters by including a more complete set of data available from the public databases and performing more 
comprehensive comparability study.

\section{Methodology}

This study acquired both pre-mVA and control signals from Physionet databases. After pre-processing, eight statistical features were extracted from the signals using box counting method of phase space reconstruction (3). Multiple series of features were passed through their respective maximum thresholds for classification into 'pre-mVA' or 'normal' signal. Performance evaluation was carried out thrice, for CUDB versus PhysikalischTechnische Bundesanstalt Diagnostic ECG Database (PTBDB), SDDB versus NSRDB, as well as CUDB and SDDB versus PTBDB and NSRDB.

\subsection{Signal Acquisition}

Pre-mVA signals were extracted from CUDB and SDDB while control signals were extracted from PTBDB and NSRDB. CUDB comprised 35 eight-minute ECG records from subjects who experienced sustained VT and VF. Record 'CU21', 'CU33' and 'CU35' were claimed to be corrupted with excessive signal noises (3) and hence discarded in our study. SDDB comprised 23 24-hours ECG records from subjects who experienced sustained ventricular arrhythmia and our study excluded three SDDB records without annotation of VF onset time ('40', '42', '49'). PTBDB contained 52 two-minutes ECG records from healthy subjects and NSRDB comprised 18 24-hours ECG records from subjects without significant arrhythmia. All PTBDB and NSRDB records were included in analysis.

For prediction purpose, ECG signal before $\mathrm{mVA}$ onset was extracted from each record of CUDB and SDDB before being used for analysis. Only lead I signal of each subject was utilized in our study to ensure the independence of signal samples. ECG records in SDDB lasted around 24 hours and only 10-minute ECG signal before onset of each record was used in this study for feature examination. ECG records in CUDB lasted only about eight minutes, therefore, the whole signal before onset of each record was used in this study. Lead I signal from each PTBDB record was chosen for analysis in this work to enable just comparison with the previous study (3). ECG records in NSRDB lasted around 24 hours and only 10-minute ECG signal of each record, starting from the first annotated normal beats after QRS-like artefacts, was used in this study for feature examination. Standard annotations in the database were used to identify the mVA onset in CUDB and QRS-like artefacts in NSRDB.

\subsection{Signal Preprocessing}

ECG signals in CUDB, SDDB, NSRDB and PTBDB were originally sampled at frequency of $250 \mathrm{~Hz}, 250 \mathrm{~Hz}$, $128 \mathrm{~Hz}$ and $1000 \mathrm{~Hz}$ respectively. Signals in CUDB, SDDB and NSRDB were upsampled to $1000 \mathrm{~Hz}$ to warrant fair comparison. Afterwards, the ECG signals were filtered using fourth-order Butterworth filter with passband ranging from $1 \mathrm{~Hz}$ to $30 \mathrm{~Hz}$ (3) to remove highfrequency measurement noise and baseline wandering. Hamilton-Tompkins algorithm was used to detect $\mathrm{R}$ peaks in the filtered signals. A window of 10 successive RR segments was chosen to construct a phase portrait of ECG signals.

\subsection{Feature Extraction}

By inserting $k$ time delay $(\tau)$ to a time series data, a $k$ dimensional phase space diagram was constructed (10). Two-dimensional phase space diagram, named phase portrait, was used for the subsequent analysis. Box counting was utilized to extract information from phase portrait, whereby box count gave an estimation of signal complexity. Statistical and nonlinear analysis of box count were performed to characterise the complexity and to identify the underlying desynchronization phenomenon of ECG signal.

In this work, ECG signals in each window were normalized to amplitude range [0,1] and a 20-ms delay was applied to obtain a total of 10 trajectories in phase portraits. The phase portrait was outputted as a 1024-by1024 high-resolution grey-scale image (Figure 1). Once the phase portrait was constructed, box counting was employed whereby the pixels through which at least one trajectory had passed were regarded as boxes and the others were not. A sliding window of $10 \mathrm{RR}$ segments was moved consecutively by one RR segment throughout the whole record (Figure 2) and this resulted in a series of box count for each record. Feature extraction was carried out on each window of 25 successive box count visited by trajectories. A sliding window of 25 box count was moved consecutively by one box count throughout the whole record and this resulted in multiple series of features for each record. Eight statistical features were calculated for each record. The statistical features included mean, standard deviation, interquartile range, mean absolute deviation, coefficient of variation (CV), coefficient of mean absolute deviation (CMAD), skewness and kurtosis of box counts.

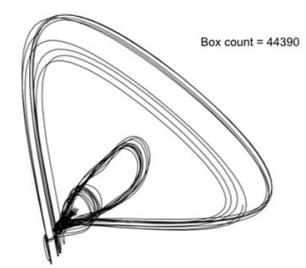

Figure 1. Each window of $10 \mathrm{RR}$ segments resulted in one phase portrait and one box count. 


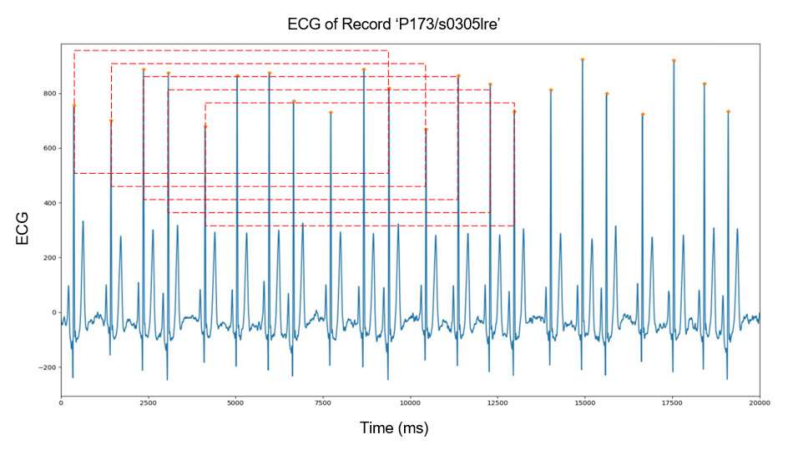

Figure 2. Sliding windows of $10 \mathrm{RR}$ segments along the time series ECG signal of record 'P173/s03051re'.

\subsection{Classification and Performance Evaluation}

For each feature, the maximum values were extracted and used to construct a Receiver Operating Characteristics (ROC) curve. The value located nearest to the upper left corner of ROC curve was chosen as the upper bound threshold because it gave high and balanced sensitivity and specificity. The optimal threshold was derived from ten-fold cross validation of ROC analysis. In this study, eight features were extracted and hence eight optimal thresholds were derived. Then, the whole time series of feature values for each record was passed through their respective optimal thresholds. Once the threshold was exceeded, the record signal was categorized as 'going-tomVA' signal, otherwise the signal was deemed as 'normal'. The ability of the selected features to distinguish between pre-mVA and control signals were assessed by means of accuracy, sensitivity, specificity and prediction time.

\section{Results}

Box count CV and CMAD achieved the highest accuracy in our study and hence were selected to be benchmarked against the two existing groups of works (CUDB and SDDB groups), followed by a final performance comparison with another study (2) using multiple databases. For each benchmarking, only the study which reported the best performance was selected. It is important to note that only 32 PTBDB records were included in the first comparative analysis to enable a fair comparison with the previous work (3). As shown in Table 1, our work attained similar performance despite using only a single feature, $\mathrm{CV}$ or $\mathrm{CMAD}$, and provided an earlier prediction time. Between $\mathrm{CV}$ and $\mathrm{CMAD}, \mathrm{CV}$ showed a better prediction time but required more complex computation.

Besides the highest performance, the selection criteria for benchmarking study in SDDB group included: 1) all
18 NSRDB records were used, 2) all 20 SDDB records were used with only a single channel from each record used for the classification and 3) the prediction time was less than 10 minutes (because our study only extracted and tested 10-minutes signal segments in this study). As shown in Table 2, again our work could attain similar performance with only a single feature, CV or CMAD. Our study achieved better average prediction time while (5) reported a constant prediction time of three minutes which was also important for clinical practice.

For the multiple database benchmarking, the result of $\mathrm{CV}$ and CMAD from our study were compared against (2) in Table 3 and our work reported slightly higher accuracy and specificity. Although the previous study reported a slightly higher sensitivity, only 18 CUDB records were used in their study without stating the reasons for inclusion or exclusion, nor specifying the records used for analysis. In contrast, our study clearly specified the records used for analysis and a more balanced data sets were used, in which the mVA-tocontrol record ratio was $43 \%$ to $57 \%$ instead of the $31 \%$ and $69 \%$ in (2). Additionally, our study also produced a much better performance compared to (9) which included similar databases for analysis.

Table 1. Prediction performance on CUDB and PTBDB.

\begin{tabular}{|c|c|c|}
\hline Study & Our Study & $\operatorname{Ref}(3)$ \\
\hline Method & Modified PSR method & PSR method \\
\hline Feature & CMAD & $\begin{array}{c}\mathrm{CV} \text { and kurtosis } \\
\text { of box count }\end{array}$ \\
\hline $\begin{array}{l}\text { Database } \\
\text { (Records } \\
\text { Number) }\end{array}$ & \multicolumn{2}{|c|}{ CUDB (32), PTBDB (32) } \\
\hline Accuracy & $98.4 \%$ & $98.4 \%$ \\
\hline Sensitivity & $100.0 \%$ & $96.9 \%$ \\
\hline Specificity & $96.9 \%$ & $100.0 \%$ \\
\hline $\begin{array}{l}\text { Prediction } \\
\text { Time }\end{array}$ & $\begin{array}{c}4 \mathrm{~m} 46 \mathrm{~s} \\
( \pm 2 \mathrm{~m} 8 \mathrm{~s})\end{array}$ & $\begin{array}{c}4 \mathrm{~m} 31 \mathrm{~s} \\
( \pm 2 \mathrm{~m} 30 \mathrm{~s})\end{array}$ \\
\hline
\end{tabular}

Table 2. Prediction performance on SDDB and NSRDB.

\begin{tabular}{lcc}
\hline Study & Our Study & Ref (5) \\
\hline Method & $\begin{array}{c}\text { Modified PSR method } \\
\text { with maximum } \\
\text { thresholding }\end{array}$ & $\begin{array}{c}\text { ECG nonlinear } \\
\text { method with } \\
\text { SVM }\end{array}$ \\
\hline Feature & CV CMAD & $\begin{array}{c}\text { Hjorth } \\
\text { parameters, } \\
\text { wavelet band } \\
\text { energy, fuzzy } \\
\text { entropy }\end{array}$ \\
\hline $\begin{array}{l}\text { Database } \\
\text { Records }\end{array}$ & SDDB (20), NSRDB (18) \\
Number) & & \\
\hline Accuracy & $94.7 \%$ & $94.7 \%$ \\
\hline
\end{tabular}




\begin{tabular}{|c|c|c|c|}
\hline Sensitivity & \multicolumn{2}{|c|}{$90.0 \%$} & $95.0 \%$ \\
\hline Specificity & \multicolumn{2}{|c|}{$100.0 \%$} & $94.4 \%$ \\
\hline $\begin{array}{l}\text { Prediction } \\
\text { Time }\end{array}$ & $\begin{array}{c}5 \mathrm{~m} 26 \mathrm{~s} \\
( \pm 3 \mathrm{~m} 46 \mathrm{~s})\end{array}$ & $\begin{array}{c}4 \mathrm{~m} 46 \mathrm{~s} \\
( \pm 2 \mathrm{~m} 8 \mathrm{~s})\end{array}$ & $3 \mathrm{~m}$ \\
\hline
\end{tabular}

Table 3. Prediction performance on CUDB and PTBDB.

\begin{tabular}{|c|c|c|}
\hline Study & Our Study & $\operatorname{Ref}(2)$ \\
\hline Method & $\begin{array}{l}\text { Modified PSR method } \\
\text { with maximum } \\
\text { thresholding }\end{array}$ & $\begin{array}{c}\mathrm{T} \text { wave } \\
\text { complexity } \\
\text { analysis with } \\
\text { maximum } \\
\text { thresholding }\end{array}$ \\
\hline Feature & CMAD & $\begin{array}{l}\text { Approximate } \\
\text { entropy }\end{array}$ \\
\hline $\begin{array}{l}\text { Database } \\
\text { (Records } \\
\text { Number) }\end{array}$ & $\begin{array}{c}\text { CUDB (32), SDDB } \\
(20), \text { PTBDB (52), } \\
\text { NSRDB (18) }\end{array}$ & $\begin{array}{c}\text { CUDB (18), } \\
\text { NSRDB and } \\
\text { CINC } \\
\text { Challenge } \\
\text { database (40) }\end{array}$ \\
\hline Accuracy & $94.3 \%$ & $93.1 \%$ \\
\hline Sensitivity & $88.5 \%$ & $88.9 \%$ \\
\hline Specificity & $98.6 \%$ & $95.0 \%$ \\
\hline $\begin{array}{l}\text { Prediction } \\
\text { Time }\end{array}$ & $\begin{array}{cc}4 \mathrm{~m} 11 \mathrm{~s} \\
( \pm 3 \mathrm{~m} 12 \mathrm{~s})\end{array} \quad \begin{array}{c}4 \mathrm{~m} 4 \mathrm{~s} \\
( \pm 3 \mathrm{~m} 9 \mathrm{~s})\end{array}$ & - \\
\hline
\end{tabular}

\section{Conclusion}

To our knowledge, our work is the first to validate an mVA prediction algorithm across two clusters of prediction work besides coming up with the final performance evaluation on the combined data sets from multiple databases. This systematic performance evaluation approach, together with analysis using complete data sets, is recommended for future research to promote the algorithm comparability. Besides, combination of PSR box count CMAD feature and maximum thresholding classification in our study achieves a better prediction time (over 4 minutes) and high-performance accuracy (over 90\%) compared to other studies. Due to the low computational effort, the proposed algorithm is potentially useful for continuous monitoring of individuals, so that users can be alerted earlier to the problem and hence have higher chances of getting treatment in time. Low computational cost typically means lower power consumption, making the algorithm suitable for embedded or wearable devices.

\section{Acknowledgments}

This work was supported by CREST R\&D grant T20C3-14 (4B244), UTM Matching Grant No. Q.J13000.3001.01M13 and UTM Grant No. Q.J130000.2523.16H47.

\section{References}

1. Mehra R. Global public health problem of sudden cardiac death. J Electrocardiol. 2007 Nov 1;40(6 SUPPL. 1):S118-22.

2. Riasi A, Mohebbi M. Prediction of ventricular fibrillation using complexity analysis of $\mathrm{T}$ Wave from surface electrocardiogram. Modares $\mathrm{J}$ Electr Eng. 2016;13(1).

3. Koulaouzidis G, Das S, Cappiello G, Mazomenos EB, Maharatna K, Puddu PE, et al. Prompt and accurate diagnosis of ventricular arrhythmias with a novel index based on phase space reconstruction of ECG. Int $\mathrm{J}$ Cardiol. 2015 Mar 1;182(C):38-43.

4. Amezquita-Sanchez JP, Valtierra-Rodriguez M, Adeli H, Perez-Ramirez CA. A Novel Wavelet TransformHomogeneity Model for Sudden Cardiac Death Prediction Using ECG Signals. J Med Syst. 2018 Oct 16;42(10):176.

5. Fujita H, Acharya UR, Sudarshan VK, Ghista DN, Sree SV, Eugene LWJ, et al. Sudden cardiac death (SCD) prediction based on nonlinear heart rate variability features and SCD index. Appl Soft Comput J. 2016 Jun $1 ; 43: 510-9$

6. Lee H, Shin S-Y, Seo M, Nam G-B, Joo S. Prediction of Ventricular Tachycardia One Hour before Occurrence Using Artificial Neural Networks. Sci Rep. 2016 Aug 26;6(1):32390.

7. Wollmann CG, Gradaus R, Böcker D, Fetsch T, Hintringer $\mathrm{F}$, Hoh $\mathrm{G}$, et al. Variations of heart rate variability parameters prior to the onset of ventricular tachyarrhythmia and sinus tachycardia in ICD patients. Results from the heart rate variability analysis with automated ICDs (HAWAI) registry. Physiol Meas. 2015 May 1;36(5):1047-61.

8. Raka A, Naik G, Chai R. Computational Algorithms Underlying the Time-Based Detection of Sudden Cardiac Arrest via Electrocardiographic Markers. Appl Sci. 2017;7(9):954.

9. Santos GS. Towards short-term forecasting of ventricular tachyarrhythmias. 2006.

10. Takens F. Detecting strange attractors in turbulence. In 1981. p. 366-81. Available from: http://link.springer.com/10.1007/BFb0091924

Address for correspondence:

Wei Wei Heng.

P19A, School of Electrical Engineering, Universiti Teknologi Malaysia, 81310 Johor Bahru, Johor, Malaysia.

vivihengww@gmail.com 